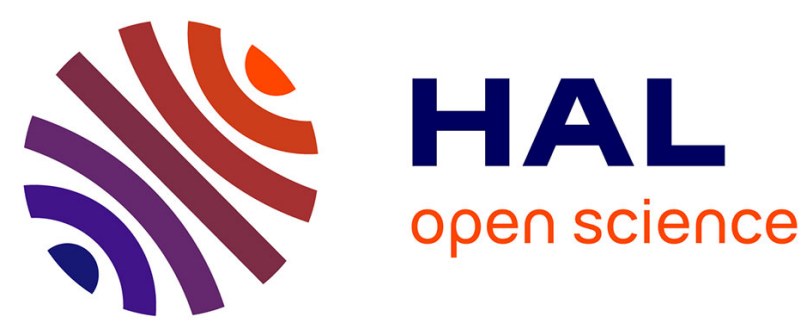

\title{
Obstetric complications as a risk factor for first psychotic episodes in childhood and adolescence
}

Dolores Moreno, Miguel Moreno-Iñiguez, Dolores Vigil, Josefina

Castro-Fornieles, Felipe Ortuño, Ana González-Pinto, Mara Parellada, Inmaculada Baeza, Soraya Otero, Montserrat Graell, et al.

\section{To cite this version:}

Dolores Moreno, Miguel Moreno-Iñiguez, Dolores Vigil, Josefina Castro-Fornieles, Felipe Ortuño, et al.. Obstetric complications as a risk factor for first psychotic episodes in childhood and adolescence. European Child and Adolescent Psychiatry, 2009, 18 (3), pp.180-184. 10.1007/s00787-008-0692-x . hal-00486567

\section{HAL Id: hal-00486567 https://hal.science/hal-00486567}

Submitted on 26 May 2010

HAL is a multi-disciplinary open access archive for the deposit and dissemination of scientific research documents, whether they are published or not. The documents may come from teaching and research institutions in France or abroad, or from public or private research centers.
L'archive ouverte pluridisciplinaire HAL, est destinée au dépôt et à la diffusion de documents scientifiques de niveau recherche, publiés ou non, émanant des établissements d'enseignement et de recherche français ou étrangers, des laboratoires publics ou privés. 
Dolores Moreno

Miguel Moreno-Iñiguez

Dolores Vigil

Josefina Castro-Fornieles

Felipe Ortuño

Ana González-Pinto

Mara Parellada

Inmaculada Baeza

Soraya Otero

Montserrat Graell

Ana Aldama

Celso Arango

Accepted: 3 March 2008

Published online: 28 January 2009

D. Moreno, MD ( $\square)$

M. Moreno-Iñiguez, MD

M. Parellada, MD - C. Arango, MD

Dept. of Psychiatry

Adolescent Unit

Hospital General Universitario Gregorio

Marañón

Calle Ibiza 43

28007 Madrid, Spain

E-Mail: lolamoreno@hggm.es

D. Vigil, MD

Epidemiology Department

Hospital General Universitario Gregorio

Marañón

Madrid, Spain

J. Castro-Fornieles, MD

Dept. of Child and Adolescent Psychiatry and Psychology

Institut Clinic of Neurosciences

IDIBAPS (Institut d'Investigacions

Biomèdiques August Pi Sunyer)

Hospital Clínic Universitari of Barcelona

Barcelona, Spain

F. Ortuño, MD

Dept. of Psychiatry and Medical

Psychology

University Clinic

College of Physicians

University of Navarra

Navarra, Spain

A. González-Pinto, MD · I. Baeza, MD

A. Aldama, MD

Stanley Institute International

Mood-Disorders Research Center

03-RC-003, Hospital Santiago Apóstol

Vitoria, Spain

\section{Obstetric complications as a risk factor for first psychotic episodes in childhood and adolescence}

Abstract There are reports of significant association between obstetric complications (OC) and childhood psychosis. Authors conducted a case-control study of 102 children and adolescents with a first episode psychosis (FEP) and 94 healthy controls (HC), using the obstetric complications scale (OCS) and their medical records, to examine the risk of FPE.

Patients were recruited from child and adolescent psychiatry units at six university hospitals and controls from publicly-funded schools of similar characteristics and from the same geographic areas. A logistic regression was performed to quantify the risk of psychosis in childhood and adolescence, based on $\mathrm{OC}$, adjusting for potential confounding factors like socio economic status (SES) and family psychiatric history (FPH). OC

\section{S. Otero, MD}

Child and Adolescent Mental Health Unit Dept. of Psychiatry and Psychology

Hospital Universitario Marqués de

Valdecilla

Santander, Spain

M. Graell, MD

Section of Child and Adolescent Psychiatry and Psychology

Hospital Infantil Universitario Niño Jesús Madrid, Spain appeared more frequently in the records of patients. Significant differences between patients and controls were found in Prenatal OC $(15.7 \%$ vs. $5.3 \%, P<0.05)$ and among them, bleeding in pregnancy showed the greatest difference between groups $(12.7 \%$ vs. $2.1 \%, P<0.01)$. In the logistic regression, bleeding in pregnancy showed a crude odds ratio (OR) of $6.7(95 \% \mathrm{CI}=1.4-30.6)$ and $5.1(\mathrm{CI}$ $95 \%=1.0-24.9)$ adjusted for SES and FPH. Therefore, bleeding in pregnancy is a likely risk factor for early-onset psychosis.

Key words early psychosis first onset - case-control OCS 


\section{Introduction}

An association between obstetric complications (OC) and schizophrenia was first mentioned in the thirties [8], but it was not until several decades later that the first reports of significant association between some specific OC (such as prematurity, toxaemia, bleeding, and severe maternal illness) and childhood psychosis were published [9]. In the fifties, the theory of "continuum of reproductive casualty" [7] stated that OC could lead to a gradient of lesions extending from foetal and neonatal death through cerebral palsy, epilepsy, mental deficiency, and behavioural disorders. After some work was conducted in the field dealing with the broad concept of OC, a cohort study was conducted in children with autism and childhood psychosis in 1975 [10], concluding that bleeding in pregnancy was associated with syndromes like autism and childhood psychosis. In that study, the tested hypothesis was that one or more perinatal events would be found to be associated with the subsequent development of infantile autism and childhood psychosis. For that purpose, authors followed a cohort of 55,908 pregnancies from physical, neurological and psychological perspectives through the 7 years of age. Among the 30,000 who completed the exams, there were records of 200 behaviourally disturbed children. Of them, 14 were selected as conforming to the syndrome of infantile autism and then matched to two control groups: one of neurologically and behaviourally normal children with low IQ and another similar one with normal IQs, matching for institution of origin (14 medical centers), race, sex, and socioeconomic factors. After this, one blind researcher took the perinatal portions of the chart and abstracted data on 26 aspects of the pre-pregnancy, pregnancy, delivery and immediate post-delivery period. The study identified a single perinatal significant event associated with autism: maternal uterine bleeding during pregnancy. Furthermore, in order to determine whether this apparent association would appear in a larger group, the authors selected, among the original 200 charts, additional children who were apparently psychotic (severely disturbed, psychotic-like, autistic or childhood schizophrenic), although not having the classical syndrome of infantile autism. Six additional children were then included in the study, and then matched with six normal IQ children. Again, the mother charts were checked for bleeding during pregnancy and it was found that three of the psychotic six subjects had history of bleeding episodes. As a conclusion, the authors stated that bleeding in pregnancy was "not only a sign of problems with the pregnancy but was also associated with a continuum of reproductive casualty, being a possible form of such casualty, syndromes like autism and childhood psychosis". After this, there have been very few studies on first psychotic episodes in children and adolescents, which seem to be important in order to study patients in the very early stages of the illness, when the information bias can be minimised [4].

The main hypothesis of the present case-control study was that the risk of presenting a first episode of psychosis (FEP) in childhood and adolescence would be higher in subjects with records of OC.

\section{Methods}

A case-control study of 102 adolescents diagnosed with a first episode psychosis (FEP) and 94 healthy controls was conducted in the context of a larger longitudinal early onset first-episode psychosis study, the child and adolescent first-episode psychosis study (CAFEPS) ([3], see this reference for further information about the methods). Patients were recruited from those who consecutively came in to the Department of Psychiatry at six different centres, in five different cities around the country, including both outpatient and inpatient facilities. The FEP group consisted of adolescents with a first psychotic episode diagnosed by a senior psychiatrist, according to both ICD-10 and DSM-IV criteria, after an assessment with the K-SADS-PL diagnostic interview [3]. The inclusion criteria for patients were age below 18 years at the time of first evaluation and presence of positive psychotic symptoms (within a psychotic episode) such as delusions or hallucinations of less than 6 months' duration since the onset of symptoms. Exclusion criteria were: presence of a concomitant Axis I disorder at the time of evaluation that might account for the psychotic symptoms (such as substance abuse, autistic spectrum disorders, post-traumatic stress disorder, or acute stress disorder), mental retardation (only if, in addition to low IQ, cognitive functioning was clinically impaired prior to onset of the disorder), pervasive developmental disorder, neurological disorders, history of head trauma with loss of consciousness, and pregnancy. Occasional substance use was not an exclusion criterion if positive symptoms persisted for more than two weeks after a negative urine drug test.

The control group was recruited from from publicly-funded schools of similar characteristics as the schools of patients and from the same geographic areas, or from children who were seen for routine pediatric visits at our hospitals. Some researchers visited the schools to explain the study. For their participation, controls were offered a coupon to buy school supplies. A trained psychologist made a 
preliminary phone screening to check for exclusion criteria. Those who passed the initial screening were interviewed with their relatives at the clinical centers by experienced child and adolescent psychiatrists. The inclusion criteria for controls were: similar age and sex and from the same geographical areas as patients, no psychiatric disorder as measured by the Kiddie-SADS-Present and Lifetime Version (K-SADS) and no neurological disorders, head trauma, pregnancy, or mental retardation (MR) (only if, besides low IQ, cognitive functioning was clinically impaired). Six controls were excluded due to diagnosis of a psychiatric disorder after the first evaluation (two attention deficits-hyperactivity disorder, three anxiety disorders, one anorexia nervosa) [3].

All the subjects and their parents signed an informed-consent prior to their participation in the study. Since this was a multi-centered project, the research design was approved by the responsible ethical committee of the six different institutions.

The Lewis-Murray scale of obstetric data (OCS) [6] was administered to parents. This is a scale administered by the clinician that retrospectively rates information on obstetric complications from medical records, birth records, and maternal interviews. It was derived from a consensus of six previous scales and consists of 15 complications with thresholds for rating them as "definite" or "equivocal" (for the purpose of this study, authors only considered the "definite" OC accounted by parents). The OC are grouped into prenatal (four items) and peri-postnatal complications (11 items). In eight of the 110 patients and four of the 98 controls comprising the original CAFEPS study population [3], it was not possible to administer the scale, resulting in the final number of 102 subjects in the FEP group and 94 in the control group. The causes why they could not be assessed were either that they were adopted children and did not live with their biological parents (including foster care), or the fact that there was no obstetric record in their charts. The charts consulted included clinical as well as obstetric records, either reported by their parents or registered by a clinician.

Socio-demographic variables and Family Psychiatric History (FPH) of any psychiatric disorder were also assessed during a specific interview with an expert psychiatrist. For the analysis, socio-economic status (SES) was assessed using the five-level HollingsheadRedlich model [5], regrouping them into three levels as follows: low (levels I and II), intermediate (level III), and high (levels IV and V).

\section{Statistical analysis}

Between-group comparisons were performed using a chi square or the Fisher exact test. Univariate and multi- variate logistic regression models (ENTER method) were used to quantify the risk of psychosis in adolescence, based on OC, adjusting for potential confounding factors such as SES and FPH.

Results are expressed as percentages (\%), $P$, odds ratios (OR), and their corresponding 95\% confidence intervals (95\% CI).

\section{Results}

The mean age was $15.65(95 \% \mathrm{CI}=15.33-15.97)$ years for the FEP group and $15.21(95 \% \mathrm{CI}=14.82-15.61)$ years for the control group. In terms of gender, males constituted $62 \%$ of the control group and $74 \%$ of the FEP group $\left(\chi^{2}=0.57 ; P=0.448\right)$. The diagnostic spectra in the FEP group comprised Psychotic Disorder Not Otherwisw Specified (35.4\%); Schizophreniform Disorder (23.6\%); Depressive Disorder With Psychotic Symptoms (11.8\%); Bipolar Disorder, Manic Episode With Psychotic Symptoms (10.9\%); Schizophrenia (10\%), Schizoaffective Disorder (2.7\%) and Other Psychotic Disorders (5.4). More specific descriptive data are showed in Table 1 (for further details, please go to [3]).

In general, OC appeared more frequently in the records of patients as compared to controls, although this difference did not reach statistical significance (34.3\% vs. $22.3 \%, P=0.06)$. (See Table 2 ). Regarding the number of OC per subject, $77 \%$ of controls and $66 \%$ of FEP subjects presented with no OC; $16 \%$ of

Table 1 Distribution by age, socioeconomic status, sex and race of patients and controls (from CAFEPS, [3])

\begin{tabular}{lll}
\hline & Controls & FEP \\
\hline Age (years) $\left(\chi^{2}=7.7 ; P=0.463\right)$ & & \\
9 & $2(2.0 \%)$ & $1(0.9 \%)$ \\
10 & $2(2.0 \%)$ & $0(0 \%)$ \\
11 & $2(2.0 \%)$ & $4(3.6 \%)$ \\
12 & $3(3.1 \%)$ & $4(3.6 \%)$ \\
13 & $8(8.2 \%)$ & $4(3.6 \%)$ \\
14 & $10(10.2 \%)$ & $13(11.8 \%)$ \\
15 & $19(19.4 \%)$ & $17(15.5 \%)$ \\
16 & $22(22.4 \%)$ & $21(19.1 \%)$ \\
17 & $30(30.6 \%)$ & $46(41.8 \%)$ \\
Socioeconomic status $\left(\chi^{2}=11.9 ; P=0.018\right)$ & \\
5 (lowest) & $11(11.2 \%)$ & $24(21.8 \%)$ \\
4 & $23(13.5 \%)$ & $36(32.7 \%)$ \\
3 & $26(26.5 \%)$ & $24(21.8 \%)$ \\
2 & $10(10.2 \%)$ & $12(10.9 \%)$ \\
1 (highest) & $28(28.6 \%)$ & $14(12.7 \%)$ \\
Sex $\left(\chi^{2}=0.57 ; P=0.448\right)$ & & \\
Males & $61(62.2 \%)$ & $74(67.3 \%)$ \\
Race $\left(\chi^{2}=4.2 ; P=0.125\right)$ & & \\
Caucasian & $91(92.8 \%)$ & $94(85.5 \%)$ \\
Hispanic & $5(5.4 \%)$ & $7(6.4 \%)$ \\
Others & $2(2.0 \%)$ & $9(8.1 \%)$ \\
\hline
\end{tabular}


Table 2 Number of reported cases for each individual obstetric complication in both groups (percentages)

\begin{tabular}{llll}
\hline Chronological OC & Obstetric complication & Control group (48\%) & FEP $^{*}$ group (52\%) \\
\hline Pre-natal OC & & & \\
& 1. Measles or syphilis & $0(0)$ & $4(100)$ \\
& 2. Rhesus incompatibility & $2(28.6)$ & $5(71.4)$ \\
& 3. Severe preeclampsia & $1(14.3)$ & $6(85.7)$ \\
& 4. Bleeding in pregnancy & $2(13.3)$ & $13(86.7)$ \\
5. Membranes rupture (more than 24 h) & $3(27.3)$ & $8(72.7)$ \\
Peri-post-natal OC & 6. Delivery duration (longer than 36 h & $3(33.3)$ & $6(66.7)$ \\
& or shorter than 3 h) & & $5(100)$ \\
& 7. Multiparity and complex delivery & $0(0)$ & $6(85.7)$ \\
& 8. Cord prolapse & $1(14.3)$ & $12(70.6)$ \\
& 9. Gestational age $<37$ o $>$ 42 weeks & $5(29.4)$ & $13(61.9)$ \\
& 10. Emergency cesarean section & $8(38.1)$ & $6(60)$ \\
& 11. Abnormal presentation & $4(40)$ & $9(81.8)$ \\
12. Use of forceps & $2(18.2)$ & $6(85.7)$ \\
13. Birthweight < 2,000 g & $1(14.3)$ & $6(100)$ \\
Total & 14. Incubator $>4$ weeks & $0(0)$ & $1(100)$ \\
& 15. Important physical anomaly & $0(0)$ & $106(76.8)$ \\
\hline
\end{tabular}

${ }^{*}$ FEP first episode psychosis controls and $20 \%$ of the FEP subjects presented with only 1 OC; $4 \%$ of controls and $7 \%$ of FEP subjects presented with two OC; and finally, $3 \%$ of controls and $7 \%$ of FEP subjects presented with more than two OC $\left(\chi^{2}=3.32 ; P=0.07\right)$.

From a chronological perspective, significant differences between patients and controls were found only in the Prenatal OC subgroup $(15.7 \%$ vs. $5.3 \%$, $P<0.05)$ and, of the Prenatal OC, bleeding in pregnancy (item 4) showed the greatest difference between groups $(12.7 \%$ vs. $2.1 \%, P<0.01)$.

In the univariate logistic regression model, bleeding in pregnancy (item 4 on the scale) showed an OR of 6.7 (95\% CI $=1.4-30.6)$. Two potential confounding factors, SES and FPH, were also explored in the analysis because of their possible influence on the results. In the multivariate analysis, they appeared as three independent variables. After adjusting for SES and FPH, the OR changed to 5.1 (95\% CI $=1.0-24.9)$. See Table 3.

\section{Discussion}

This study was conducted in the context of the CAFEPS [3], to our knowledge, the largest early-onset first-episode psychosis sample ever studied and the one with the shortest duration of symptoms and psychopharmacological treatment. According to the present results, there is a significant association between bleeding during pregnancy and first psychotic episodes in adolescence (adjusted OR $>5$ ). This finding tends to corroborate previous studies [10]. The present study is a case-control whereas the above mentioned one was a cohort study. However, in this case the sample size was larger, the overall assessment was based on a validated scale [6], and diagnoses were based on more restrictive diagnostic criteria. Furthermore, following the recommendations of previous studies, the risk measure was controlled for two likely confounding variables, SES and FPH, because of their

Table 3 Risk factors associated with first psychotic episodes in children and adolescents

\begin{tabular}{|c|c|c|c|c|c|c|c|c|}
\hline \multirow[t]{2}{*}{ Factors } & \multirow[t]{2}{*}{$O R^{a}$} & \multicolumn{2}{|c|}{$95 \% \mathrm{Cl}^{\mathrm{a}}$} & \multirow[t]{2}{*}{$P^{a}$} & \multirow[t]{2}{*}{$O R^{b}$} & \multicolumn{2}{|c|}{$95 \% \mathrm{Cl}^{\mathrm{b}}$} & \multirow[t]{2}{*}{$P^{\mathrm{b}}$} \\
\hline & & Lower & Upper & & & Lower & Upper & \\
\hline Bleeding in pregnancy & 6.72 & 1.47 & 30.63 & $<0.05$ & 5.01 & 1.01 & 24.95 & $<0.05$ \\
\hline $\mathrm{FPH}^{1}$ & 4.94 & 2.59 & 9.41 & $<0.001$ & 4.25 & 2.174 & 8.321 & $<0.001$ \\
\hline \multicolumn{9}{|l|}{$\mathrm{SES}^{2}$} \\
\hline High & 1 & - & - & - & - & - & - & - \\
\hline Intermediate & 1.28 & 0.59 & 2.70 & 0.52 & 1.35 & 0.58 & 3.12 & 0.48 \\
\hline Low & 3.30 & 1.67 & 6.50 & $<0.005$ & 3.11 & 1.48 & 6.50 & $<0.01$ \\
\hline
\end{tabular}

${ }^{1} F P H$ family psychiatric history, ${ }^{2}$ SES Socio-economic status

${ }^{a}$ Crude odds ratio (OR), 95\% confidence intervals $(\mathrm{Cl})$, and statistical significance $(P)$. Univariate logistic regression analysis

${ }^{\mathrm{b}}$ Adjusted odds ratio (OR), 95\% confidence intervals $(\mathrm{Cl})$, and statistical significance $(P)$. Multivariate logistic regression analysis 
potential influence on the results [2]. After this adjustment, bleeding in pregnancy still appeared as a significant risk factor, pointing out its association with FEP in childhood and adolescence. Further research is needed, especially cohort studies of high risk populations, in order to corroborate this results more adequately. Besides, further studies should not focus only on this factor but also on other OCs that did not result statistically significant in the present study, partly due to the low rates detected of some of them. Thus, like in previous studies [10], it is difficult to state that other complications are not related to higher risk, as the reader can note in Table 2, where the frequency of obstetric complications is higher for all of the 15 items.

Among the limitations of the present study, it is important to bear in mind that retrospective assessment of OC, as measured by an interview, can introduce a recall bias, even though information was supplemented with pregnancy/delivery medical records when possible. When completing the OCS scale, authors always asked the participant's parents or legal tutors for the clinical records, in order to get a more reliable fund of information, and it was possible in some cases but not in all of them. Unfortunately, the final number of patients and controls that did not have their records completed with clinical records was not registered and is not available. Anyway, as explained in the methods section, those participant's whose parents or legal tutors did not constitute a reliable fund of information and were unable to respond any of the items, were not considered in the present study. Since findings were not adjusted for multiple comparisons, there is a chance for Error type I. Besides, given the low rates of OCs, the present study is likely underpowered to detect significant differences for many of the items. Furthermore, it is important to take into account that, in a clinical interview involving long-term recall, women will accurately report only important medical events taking place around pregnancy and delivery [1]. Thus, bleeding in pregnancy may be an easy recallable event for mothers, even 9-17 years after the fact.

In summary, prenatal OC seem to be more common in the records of patients and, of those OC, bleeding in pregnancy may constitute a significant risk factor for first psychotic episodes in childhood and adolescence.

Acknowledgments "CIBER 07/09, CIBER de Salud Mental (CIBERSAM). Supported by the Spanish Ministry of Health, Instituto de Salud Carlos III". This work was presented as a poster at the 2007 International Congress on Schizophrenia Research, in Colorado Springs (Colorado, USA), which took place between March 28 and April 2, 2007.

\section{References}

1. Buka SL, Goldstein JM, Spartos E, Tsuang MT (2004) The retrospective measurement of prenatal and perinatal events: accuracy of maternal recall. Schizophr Res 71(2/3):417-426

2. Cannon M, Jones PB, Murray RM (2002) Obstetric complications and schizophrenia: historical and metaanalytic review. Am J Psychiatry 159(7):1080-1092

3. Castro-Fornieles J, Parellada M, Gonzalez-Pinto A et al (2007) The child and adolescent first-episode psychosis study (CAFEPS): design and baseline results. Schizophr Res 91(1-3):226-237
4. Geddes JR, Lawrie SM (1995) Obstetric complications and schizophrenia: a meta-analysis. Br J Psychiatry 167(6):786-793

5. Hollingshead AB, Redlich FC (1954) Schizophrenia and social structure. Am J Psychiatry 110(9):695-701

6. Owen MJ, Lewis SW, Murray RM (1988) Obstetric complications and schizophrenia: a computed tomographic study. Psychol Med 18(2):331339

7. Pasamanick B, Rogers ME, Lilienfeld AM (1956) Pregnancy experience and the development of behavior disorders in children. Am J Psychiatry 112(8):613-618
8. Rosanoff AJ, Handy LM, Plesset IR et al (1934) The etiology of so-called schizophrenic psychoses: with special reference to their occurrence in twins. Am J Psychiatry 91:247-286

9. Terris M, Lapouse R, Monk MA (1964) The Relation of prematurity and previous fetal loss to childhood schizophrenia. Am J Psychiatry 121:476-481

10. Torrey EF, Hersh SP, McCabe KD (1975) Early childhood psychosis and bleeding during pregnancy. A prospective study of gravid women and their offspring. J Autism Child Schizophr 5(4):287-297 